\title{
Design of a Flexible Capture Mechanism Inspired by Sea Anemone for Non-cooperative Targets
}

Jiankun Yang ${ }^{1,2}$, Chengwei Ren ${ }^{1}$, Chenghao Yang ${ }^{1}$, Youyu Wang ${ }^{3}$, Shumin Wan ${ }^{1}$ and Rongjie Kang ${ }^{1 *}$ (1)

\begin{abstract}
Robotic grippers have been used in industry as end-effectors but are usually limited to operations in pre-defined workspace. However, few devices can capture irregularly shaped dynamic targets in space, underwater and other unstructured environments. In this paper, a novel continuum arm group mechanism inspired by the morphology and motions of sea anemones is proposed. It is able to dissipate and absorb the kinetic energy of a fast moving target in omni-direction and utilize multiple arms to wrap and lock the target without accurate positioning control. Wire-driven actuation systems are implemented in the individual continuum arms, achieving both bending motion and stiffness regulation. Through finite element method, the influence of different configurations of the continuum arm group on the capture performance is analyzed. A robotic prototype is constructed and tested, showing the presented arm group mechanism has high adaptability to capture targets with different sizes, shapes, and incident angles.
\end{abstract}

Keywords: Non-cooperative targets, Continuum arm group, Wire-driven mechanism

\section{Introduction}

Traditional grippers with rigid links and joints, usually adopt two-finger or three-finger configurations to pick and hold objects, taking the advantages of high precision and stiffness. They are suitable for operations in pre-defined workspace. For instance, Drigalski et al. [1] developed a versatile two-finger gripper that can grab ultra-thin objects such as textiles and clothing. Samavati et al. [2] presented a three-finger robotic gripper to grasp cylindrical objects via "force-closure" and "form-closure". Nie et al. [3] combined two simple grippers, including an inner one for precise alignment and an outer one for stable holding, for the use of assembly. Bolboe and Staretu [4] developed a gripper with two symmetrical fingers that can grasp objects within a certain size range by placing sensors on the articulated modules. Humanoid robotic

\footnotetext{
${ }^{*}$ Correspondence: rjkang@tju.edu.cn

${ }^{1}$ Key Laboratory of Mechanism Theory and Equipment Design of Ministry of Education, Tianjin University, Tianjin 300350, China

Full list of author information is available at the end of the article
}

hand is an improvement for traditional gripper with high degrees of freedom (DOF), and can therefore provide more delicate operations through the coordination of multiple fingers. Butterfass et al. [5] developed the DLR-Hand II with four fingers, which can achieve precise grasp and fine manipulation. Odhner et al. [6] presented the iHY hand capable of in-hand repositioning tasks. Dai et al. [7] presented a metamorphic hand that has augmented orientation and workspace. Note that, existing robotic grippers and hands were mostly designed to deal with objects that remain still or move in a given direction and relatively low speed, e.g., the parts on an industrial assembly line.

With the developments of robotic technologies, human beings are expanding their exploration field to unknown environments, such as space and underwater, where the operation objects become non-cooperative, like satellites, space debris, marine life and wreckages. Compared with those pre-identified targets in industrial scenarios, non-cooperative ones have unknown

\section{Springer Open}

(c) The Author(s) 2021. Open Access This article is licensed under a Creative Commons Attribution 4.0 International License, which permits use, sharing, adaptation, distribution and reproduction in any medium or format, as long as you give appropriate credit to the original author(s) and the source, provide a link to the Creative Commons licence, and indicate if changes were made. The images or other third party material in this article are included in the article's Creative Commons licence, unless indicated otherwise in a credit line to the material. If material is not included in the article's Creative Commons licence and your intended use is not permitted by statutory regulation or exceeds the permitted use, you will need to obtain permission directly from the copyright holder. To view a copy of this licence, visit http://creativecommons.org/licenses/by/4.0/. 
shapes and undefined moving trajectory. In addition, they are impossible to communicate with in most cases.

Many efforts have been paid on exploring the capture technologies for non-cooperative targets. Yoshida and Nakanishi [8] presented a satellite capture device that can insert an impedance controlled probe into the thruster nozzle of the satellite. Meng et al. [9] presented a method to fully identify the inertial parameters of a non-cooperative object on orbit. Li et al. [10] found that accurate measurement of position and orientation for non-cooperative targets is of great importance and has a direct impact on the success rate of capture. Rekleitis et al. [11] proposed to use two 7-DOF manipulators for the on-orbit capture and service, and successfully performed ground tests. To access the ocean depths, Khatib et al. [12] developed the Ocean One, a bimanual force-controlled humanoid robot that affords intuitive haptic interaction in underwater environments. The above devices basically have rigid structures and adopt "point-to-point" operating mode which requires accurate calculation of the trajectory and orientation of the non-cooperative target. In the meantime, the capture devices need to take real-time adjustment of its own position and orientation to contact the fast moving targets as gentle as possible, preventing from collision and damage. These requirements are very challenging and so far there is no successful case of capturing noncooperative target in space [13].

On the contrast, it is noticed that flexible capture devices made of semi-soft or fully soft materials can provide redundant passive DOF to fit the non-cooperative targets while reducing the contact impact. Walker [14, 15] designed a series of continuum arms inspired by elephant trunks, which are capable of wrapping an object with the body structure. The presented continuum arms usually consist of several modules connected in series. Each module contains 3 to 6 pneumatic muscles arranged in parallel to achieve stretching and bending motion. It is found that the continuum robot can reduce the velocity of a non-cooperative target through structural compliance and surface friction [16]. Zhai et al. [17, 18] proposed a flexible flynet mechanism that can be catapulted to the target and envelope it. Whitesides et al. $[19,20]$ designed a variety of soft actuators and used them to achieve grasping of fragile objects such as eggs. Galloway et al. [21] presented a design of an underwater gripper that utilizes soft materials to delicately sample fragile species on the reef. Kang et al. [22-24] designed a series of compliant continuum arms, driven by pneumatic muscle and rod, for grasping tasks in complex environments. Dong et al. [25, 26] designed a series of continuum robots which can perform in-situ maintenance in an aeroengine, reducing the impact and damage.
However, the above-mentioned flexible capture mechanisms still have the following limitations: insufficient adaptability to the shape and size of the target, high requirements for position/orientation accuracy, and difficulties in capturing dynamic targets.

It has been found that the sea anemone is an amazing predator in the nature. It is soft and boneless, with a large number of cylindrical or vermicular tentacles as shown in Figure 1 [27]. Although the movement and load capacity of its single tentacle is not outstanding, multiple tentacles can be activated simultaneously to hook and wrap a prey coming from any direction, showing high flexibility and large tolerance.

In this paper, a flexible capture mechanism inspired by sea anemone is proposed, which can significantly improve the robustness of the capture operation for noncooperative targets while reducing the control difficulty. This paper is organized as follows: Section 2 describes the structural design of the capture mechanism; Section 3 analyzes the influence of the arm group configuration, as well as the velocity and size of the target body, on the capture success rate via a finite element model. Section 4 shows the experimental results, and the last section presents our conclusions.

\section{Design of the Capture Mechanism}

In order to deal with non-cooperative targets that are irregularly shaped and moving fast in omni-direction, the following requirements for the capture mechanism should be satisfied.

1) The mechanism should have high tolerance to the position and orientation errors during capture operation. In other words, it does not have to precisely adjust its arms to point to the target.

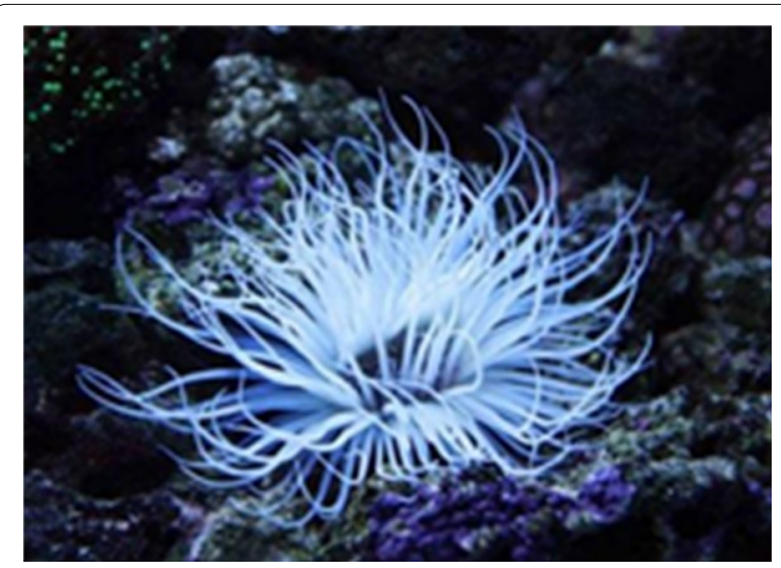

Figure 1 Sea anemone 
2) The mechanism should have appropriate compliance that can absorb the kinetic energy of the target, and therefore reduce the collision impact, as well as the damage to either the target or the capture mechanism itself.

3) The mechanism should have high adaptability to the shape and size of the target.

As mentioned in Section 1, a natural paradigm demonstrating high tolerance, compliance and adaptability in predating operation is the sea anemone. Inspired by this, we presented a capture mechanism using a number of flexible continuum arms to form an arm group to intercept and capture non-cooperative targets. The robotic prototype is composed of nine under-actuated continuum arms and a drive box, as shown in Figure 2. Parameters of the capture mechanism can be seen in Table 1 .

\subsection{Continuum Arm}

Figure 3 shows the construction of a single continuum arm, consisting of a silicone sleeve, a central skeleton, and two driving wires 1 and 2 for bending and stiffness control, respectively.

The silicone sleeve covers the central skeleton, providing the arm a soft surface to contact with a target. The central skeleton is composed of multiple segments (14 segments in our case) connected in serial by the spherical joints. The segments are made of photosensitive resin via 3D printing. As shown in Figure 3(b), each segment has a central guide hole for the routing of driving wire 2 , as well as a side guide hole which offsets from the center guide hole by $4.5 \mathrm{~mm}$ for the routing of driving wire 1 .
Table 1 Parameters of the capture mechanism

\begin{tabular}{ll}
\hline Parameter & Value \\
\hline The overall height of the capture mechanism H $(\mathrm{mm})$ & 300 \\
The overall length of the capture mechanism $L(\mathrm{~mm})$ & 140 \\
The overall width of the capture mechanism $K(\mathrm{~mm})$ & 120 \\
Height of drive box $h(\mathrm{~mm})$ & 140 \\
Length of the continuum arm / (mm) & 160 \\
The diameter of the continuum arm $\varphi(\mathrm{mm})$ & 20 \\
The thickness of the silicone sleeve $m(\mathrm{~mm})$ & 2.5 \\
Length of driving wire $1 \lambda_{1}(\mathrm{~mm})$ & 190 \\
Length of driving wire $2 \lambda_{2}(\mathrm{~mm})$ & 240 \\
Diameter of driving wire $1 \Phi_{1}(\mathrm{~mm})$ & 0.4 \\
Diameter of driving wire $2 \Phi_{2}(\mathrm{~mm})$ & 0.48 \\
The distance of adjacent segments $\delta(\mathrm{mm})$ & 9 \\
The offset distance of side guide hole $s(\mathrm{~mm})$ & 4.5 \\
Maximum rotating angle of the spherical joint $a\left(^{\circ}\right)$ & 25 \\
\hline
\end{tabular}

Both driving wires 1 and 2 are attached to the tip of the arm and go through the whole arm length to the base.

By applying a pulling force to driving wire 1 , the offset arrangement will generate a moment to the arm tip, making the arm bend to the direction of side holes. In our prototype, the direction of the side holes are arranged toward the center of the base platform of the drive box, so all arms will bend to the center of the base platform when pulling driving wire 1 . As the segments are connected by spherical joints, the arm is also allowed to passively and locally bend to other directions when touching the object. By applying a pulling force to driving wire 2,

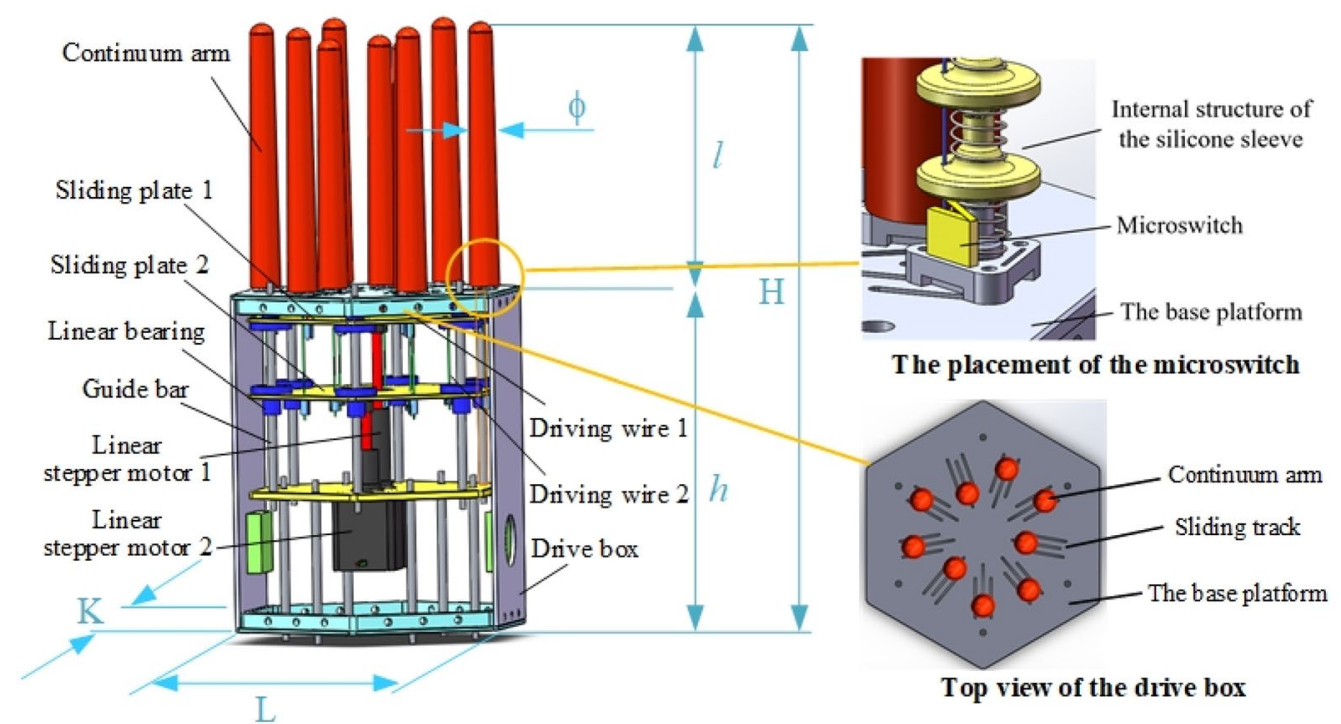

Figure 2 Design of the capture mechanism inspired by sea anemone 


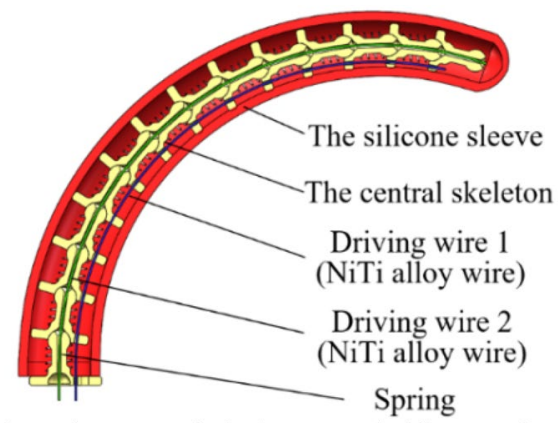

(a) The central skeleton and silicone sleeve

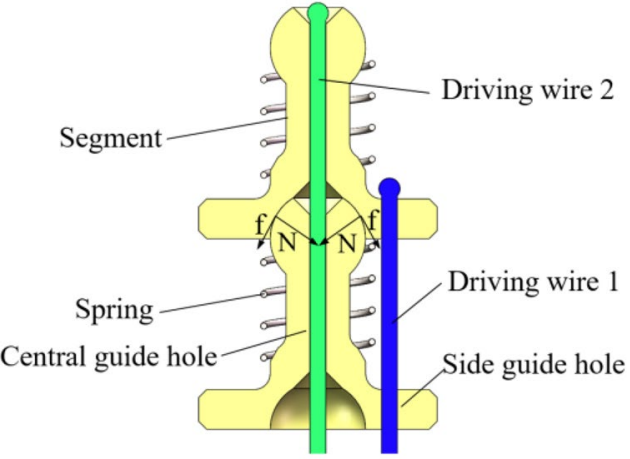

(b) Adjacent segments connected by a spherical joint

Figure 3 Structure of a single arm

the tension will increase the contacting force between individual segments and therefore limit the relative rotating motion between them, achieving the stiffness augment of the arm. Both driving wire 1 and driving wire 2 are made of NiTi alloy wire with high compliance. In addition, a spring is placed between two adjacent segments to improve the resilient force of the arm to its neutral position.

To sense the contact between the arm and an object, a microswitch is installed at the bottom of the arm. As shown in Figure 2(a). Once there is vibration on the arm, the microswitch will be activated to generate a low level signal. The base positions of the nine arms can be adjusted on the base platform of the drive box through the sliding tracks to achieve different arm configurations.

The use of arm group allows for flexible deformation to grasp target coming from a wide range of direction and position. Even if failures occur to a single continuum arm, the rest arms can still complete the task. This mechanism provides significant tolerance, compliance and adaptability required by the capture operation for non-cooperative targets.

\subsection{Drive Box}

As shown in Figure 2, the prototype includes nine continuum arms, so there are nine driving wires 1 and 2
Table 2 Parameters of the linear stepper motors

\begin{tabular}{lll}
\hline Parameter & Motor $\mathbf{1}$ & Motor $\mathbf{2}$ \\
\hline Type & $35 \mathrm{H} 4 \mathrm{~N}-05-\mathrm{A} 26$ & $43 \mathrm{M} 4 \mathrm{~B}-05-\mathrm{A} 17$ \\
Step $(\mathrm{mm})$ & 0.003048 & 0.015875 \\
Step angle $\left(^{\circ}\right)$ & 1.8 & 1.8 \\
Stroke $(\mathrm{mm})$ & 25 & 50 \\
\hline
\end{tabular}

whose bottom ends go into the drive box and fixed to two sliding plates 1 and 2, respectively. The movement of sliding plate 1 will simultaneously apply tension to all driving wires 1 and initiate a bending motion to all arms to grasp an object. Similarly, the movement of sliding plate 2 will simultaneously apply tension to all driving wires 2 and stiffen all arms. The sliding plates 1 and 2 are connected to and driven by two linear stepper motors 1 and 2 , respectively. The maximum stroke of the linear stepper motor 1 is $25 \mathrm{~mm}$, resulting a maximum bending angle of the individual continuum arms by $125^{\circ}$. Parameters of the stepper motors are given in Table 2.

\subsection{Control System}

As explained in Section 2.1, the presented prototype uses an arm group instead of a single arm to achieve high tolerance and adaptability in capture task. All arms are actuated by two stepper motors to perform bending motion and stiffness regulation, respectively. The control structure is shown in Figure 4. The computer sends the desired length of driving wires 1 and 2 to a microprocessor, Arduino (Mega2560). The Arduino processor then generates corresponding electrical pulses to the motor drivers and control the linear displacement of the stepper motors, Figure 4(a). If the microswitches detect vibration on any of the arms, the stepper motor 1 will be activated to pull the driving wires 1 , making all arms bend toward the center of the arm group to grasp the target. The stepper motor 2 will be activated to pull the driving wires 2 and stiffen the arms once stepper motor 1 reaches its maximum stroke, Figure 4(b).

\section{Finite Element Model}

This section establishes a finite element model (FEM) for the arm group mechanism to investigate the influence of group configuration, target size and velocity on the success rate of capture operation. The following assumptions are used in the model.

A single continuum arm can be simplified to an elastic rod whose bottom end fixed to the drive box while the tip end considered as a free end. 


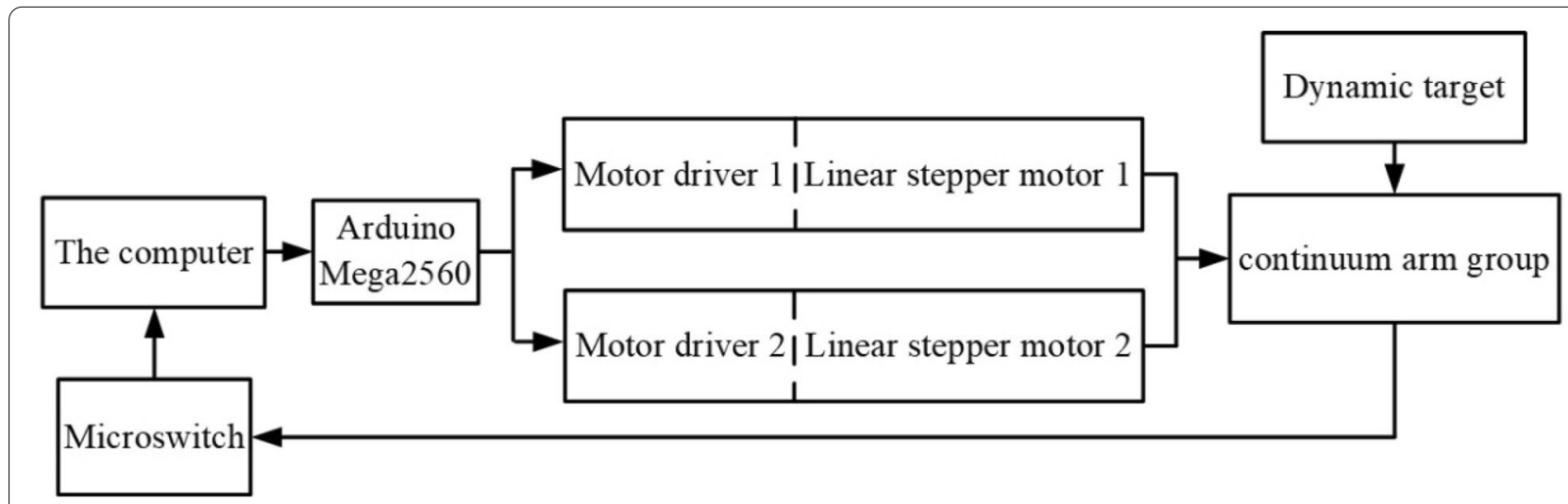

(a) Control hardware

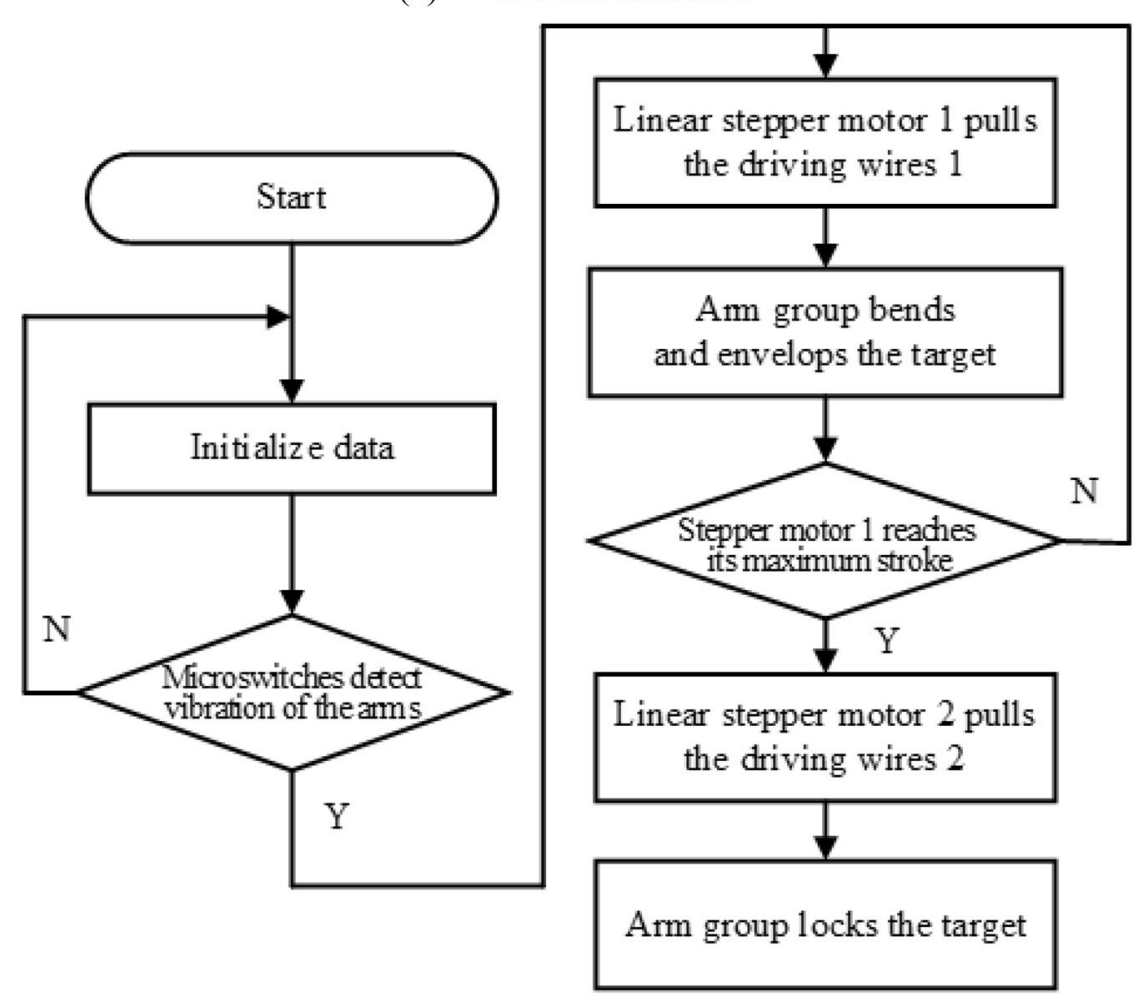

(b) Control flow chart

Figure 4 Construction of the control system

The material properties of the arm body are uniform, ignoring the internal structure of the arm.

The finite element model is established in the ABAQUS/CAE integrated environment. As mentioned above, the continuum arm is considered to be made of single material, and the equivalent elastic modulus is determined by the experiments. A DS2-50N material Tester is used to impose a transverse load on the free end of the arm to obtain the equivalent elastic modulus [28], according to

$$
\begin{aligned}
& \omega=\iint\left(\frac{M}{E I} \mathrm{~d} x\right) \mathrm{d} x+\mathrm{A} x+\mathrm{B}, \\
& \theta=\frac{\mathrm{d} \omega}{\mathrm{d} x}=\int \frac{M}{E I} \mathrm{~d} x+\mathrm{A},
\end{aligned}
$$

where $\omega$ is the deflection of the arm, representing the displacement of the arm along the direction of the transverse load, $\theta$ is the angle between the tangent line of the flexure and the vertical position of the arm. A and B are 


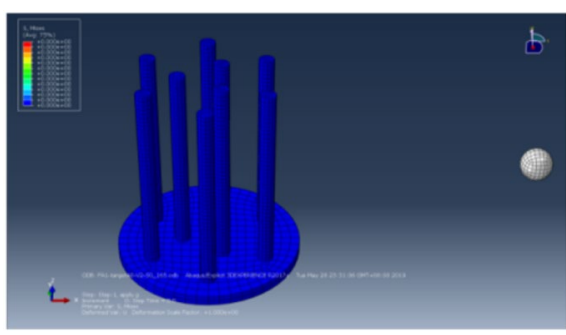

(a) Target ball is launched at initial time

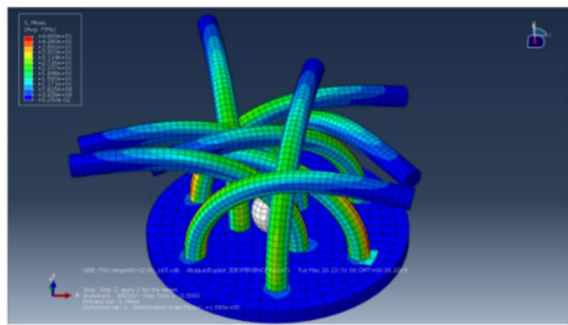

(b) Target ball is successfully captured

Figure 5 Capture of a target ball in FEM simulation

the integration constants whose values are determined by the boundary conditions. In the case of a cantilever beam, we assume $\mathrm{A}=\mathrm{B}=0 . M$ is the bending moment generated by the transverse load applied to the arm, which can be computed as the product of the transverse load and the length of the arm. $E$ is the equivalent elastic modulus of the arm. $I$ is the moment of inertia of the cross section of the arm and can be computed as $I=\frac{\pi D^{4}}{64}$, in which $D$ is the diameter of the cross section. Through Eqs. (1) and (2), the elastic modulus of the arm can be estimated as $E_{1}=3.5 \times 10^{2} \mathrm{MPa}$.

The continuum arm has a flexible body and its cross section is circular, so we can estimate the Poisson's ratio of the continuum arm according to the method of lateral compression test [29]. The obtained Poisson's ratio is $\mu_{1}=0.47$.

The element type of the continuum arm is set as C3D8R in the finite element model. The size of the element is set as $3 \mathrm{~mm}$, and the number of the elements is 10440. Also, friction is considered due to the surface contact between the continuum arms and the target. In our case, the materials used for the silicone sleeve and the target body are ELASTOSIL ${ }^{\circledR} 4601$ and Somos Imagine 8000 photosensitive resin, respectively. Thus, the static friction factor is obtained as $f_{\mu}=0.3$ by performing sliding movement experiments between these two materials.

The material of the target ball is nylon whose modulus of elasticity is $E_{2}=8.3 \times 10^{3} \mathrm{MPa}$ and Poisson's ratio is $\mu_{2}=0.28$ [30].

The output force of the linear stepper motor 2 can reach $330 \mathrm{~N}$ which is considered to be evenly applied to

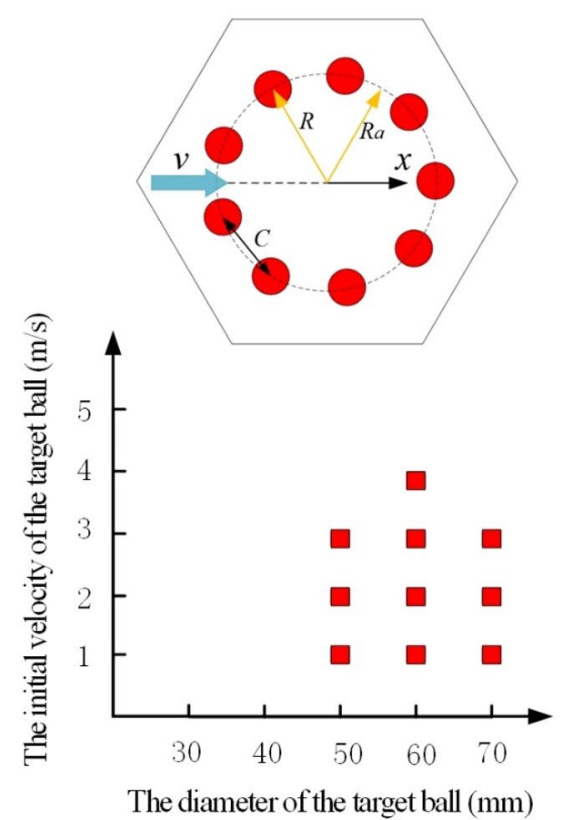

(a) $R=90 \mathrm{~mm}, R_{\mathrm{a}}=90 \mathrm{~mm}, C=61.56 \mathrm{~mm}$

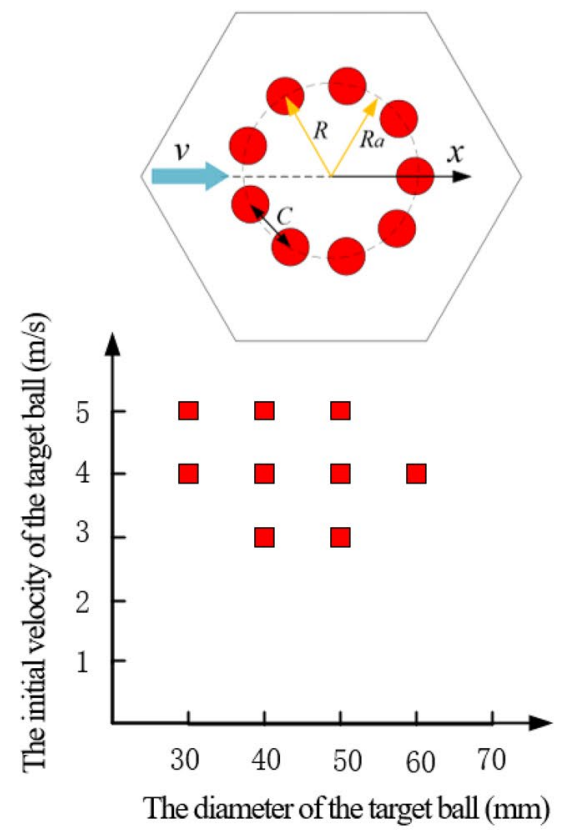

(b) $R=60 \mathrm{~mm}, R_{\mathrm{a}}=60 \mathrm{~mm}, C=41.04 \mathrm{~mm}$

Figure 6 Arms are arranged in the same circle

nine driving wires 2 . Therefore, the tension of each driving wire 2 is set to $36 \mathrm{~N}$ in the finite element model.

When $t=0 \mathrm{~s}$, a target ball with different diameters, $d$ $=30,40,50,60$ and $70 \mathrm{~mm}$, were launched to the center of the arm group with different initial speeds, $v=1,2,3$, 4 and $5 \mathrm{~m} / \mathrm{s}$, as shown in Figure 5(a). If the ball can be 


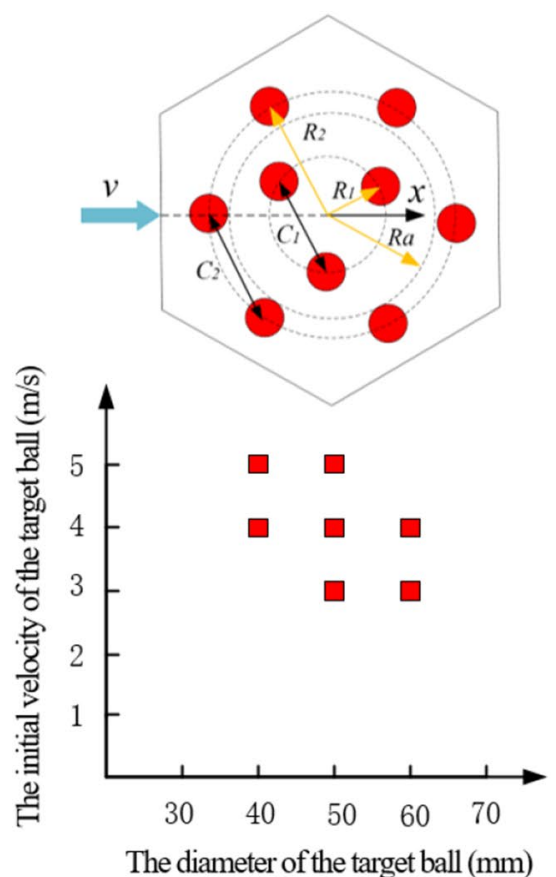

(a) $\theta=0^{\circ}$
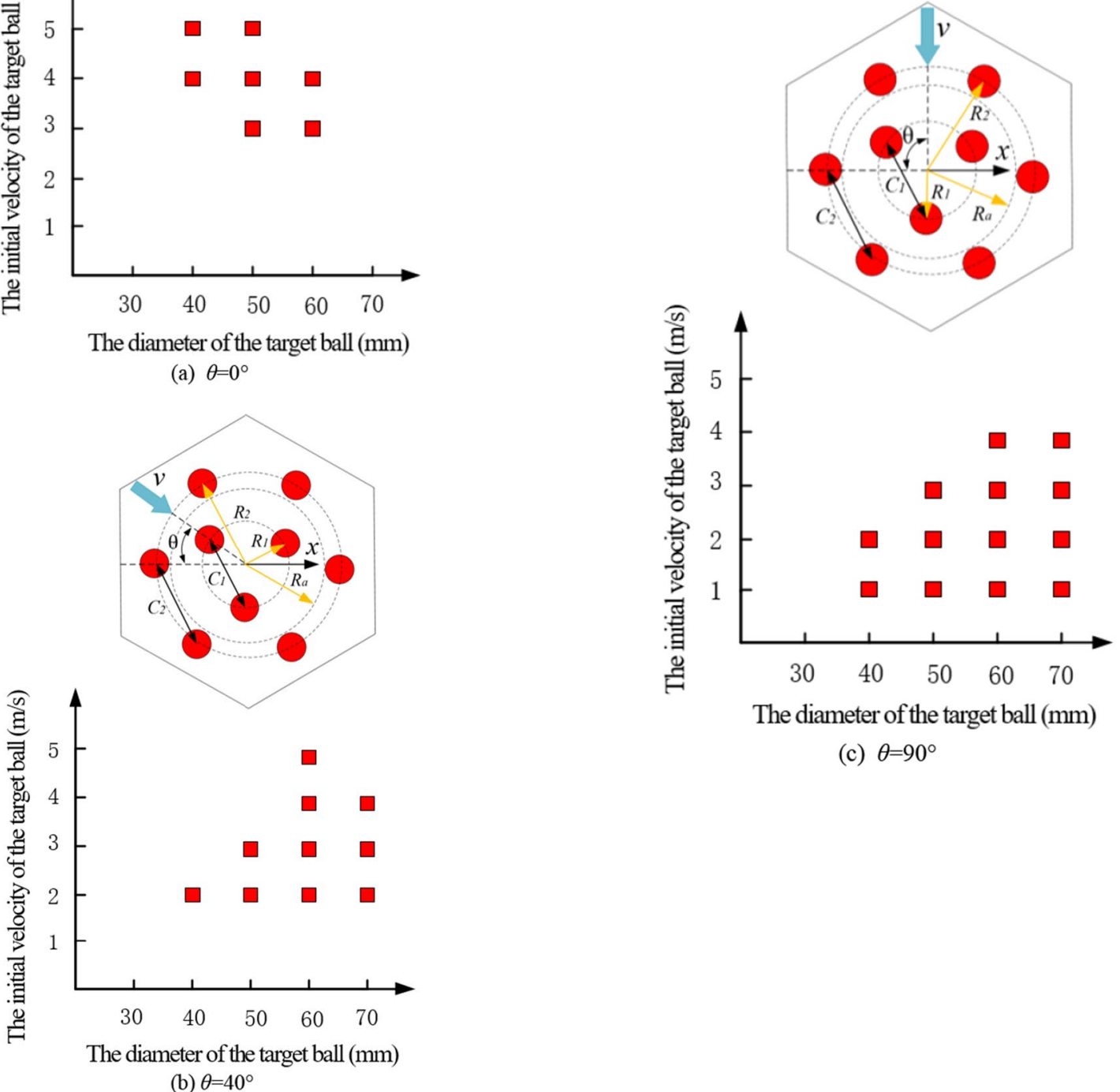

(c) $\theta=90^{\circ}$

(b) $\theta=40^{\circ}$

Figure 7 Arms are arranged in two circles where $R_{1}=30 \mathrm{~mm}, R_{2}=60 \mathrm{~mm}, R_{\mathrm{a}}=50 \mathrm{~mm}, C_{1}=51.96 \mathrm{~mm}, C_{2}=60 \mathrm{~mm}$

stopped and grasped by the arm group eventually, Figure 5(b), we record it as a successful capture.

Here, we define a concept of average distribution radius, $R_{\mathrm{a}}=\frac{\sum_{i}^{n} R_{i}}{n}$ to describe the density of the arm group, where $R_{i}(i=1,2, \ldots, 9)$ is the distance between the arm center to the group center. A large value of $R_{\mathrm{a}}$ means a low density of the arm group, and vice versa. The clearance of the arms, $C$, is defined as the linear distance of adjacent arms on the same distribution circle, as shown in Figures 6, 7 and 8.

We start the tests with a simple configuration that all arms are arranged in a circle, as shown in Figure 6. The 


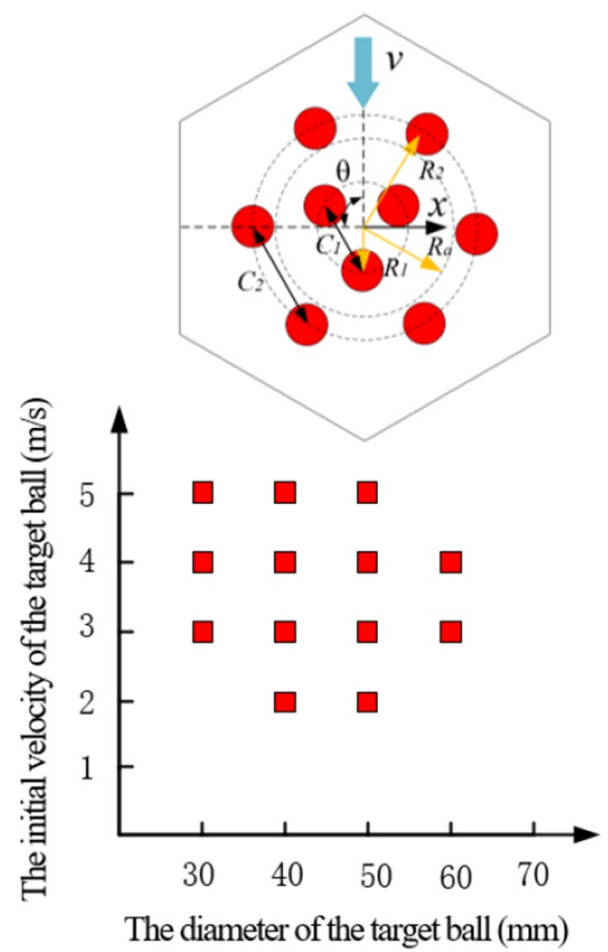

Figure 8 Arms are arranged in two circles where $R_{1}=20 \mathrm{~mm}$, $R_{2}=50 \mathrm{~mm}, R_{\mathrm{a}}=40 \mathrm{~mm}, C_{1}=34.64 \mathrm{~mm}, \mathrm{C}_{2}=50 \mathrm{~mm}$

average distribution radius in Figure 6(a) is $R_{\mathrm{a}}=90 \mathrm{~mm}$ while that in Figure $6(\mathrm{~b})$ is $R_{\mathrm{a}}=60 \mathrm{~mm}$. The diameter and velocity of the target ball resulting in successful captures are denoted by squares on a diameter-velocity plane. In Figure 6(a), the successful captures are mostly located from $d=50-70 \mathrm{~mm}$, around the clearance of the arms $C=61.6 \mathrm{~mm}$. In Figure 6(b), the range of $d$ is between $30 \mathrm{~mm}$ and $60 \mathrm{~mm}$, which is also near the clearance $C=41.0 \mathrm{~mm}$. These indicate that the success rate can be increased by making the clearance of the arms close to the diameter of the target ball. The velocity range in Figure $6(\mathrm{a})$ between $1 \mathrm{~m} / \mathrm{s}$ and $4 \mathrm{~m} / \mathrm{s}$ is lower than that in Figure 6(b) between $3 \mathrm{~m} / \mathrm{s}$ and $5 \mathrm{~m} / \mathrm{s}$. This is because the arms in Figure 6(a) has lower distribution density so it cannot provide sufficient resistance to stop the target ball with high velocity.

In addition, the continuum arms can be divided into two sets and arranged on two concentric circles with different radius, $R_{1}$ and $R_{2}$, as shown in Figure 7. Here, we set $R_{1}=30 \mathrm{~mm}$ and $R_{2}=60 \mathrm{~mm}$. We define the incident angle $\theta$ of the target ball as the sharp angle between its velocity direction and the $x$ axis. Three incident angles are then tested for this configuration. In Figure $7(\mathrm{a})$, if the incident angle is $0^{\circ}$, the target ball will firstly hit the leftmost arm. In Figure 7(b), the incident angle is $40^{\circ}$, and the target ball will contact with three continuum arms at the beginning of collision. When the incident angle is $90^{\circ}$, the target body can contact with 5 arms at the beginning of collision, Figure $7(\mathrm{c})$. As a result, there are 7, 10 and 13 successful capture points in Figure 7(a), (b) and (c), respectively. This is because the incident angle in Figure $7(\mathrm{c})$ can make more arms to contact with the target when the collision occurs.

Based on the arm group configuration shown in Figure $7(\mathrm{c})$, we reduce the average distribution radius of the arms from $50 \mathrm{~mm}$ to $40 \mathrm{~mm}$ to carry out the simulation. The results are plotted in Figure 8. Compared with Figure $7(\mathrm{c})$ where the successful capture points are located in the area of $d \in[40 \mathrm{~mm}, 70 \mathrm{~mm}]$ and $v \in[1 \mathrm{~m} / \mathrm{s}, 4 \mathrm{~m} / \mathrm{s}]$, the successful capture points in Figure 8 moves to a new area where the target ball has lower diameter $d \in[30 \mathrm{~mm}$, $60 \mathrm{~mm}]$, but higher velocity $v \in[2 \mathrm{~m} / \mathrm{s}, 5 \mathrm{~m} / \mathrm{s}]$. These phenomena are consistent with the conclusions obtained in Figure 6.

From the above analysis, we can summarize the following three conclusions:

1) The success rate of the capture can be improved by setting an appropriate average distribution radius, making the clearance of the arms close to the diameter of the target ball.

2) The incident angle of the target ball has an impact on the capture performance. The incident angle which makes more arms to contact with the target ball can help to absorb the kinetic energy and improve the success rate.

3) The density of the arm group also affects the capture performance. Increasing the density of the arm group can improve the capture ability of the target body with high velocity, and vice versa.

According to the simulated results, we implement the configuration shown in Figure 8 to our prototype to achieve high success rate because the dynamic target used in the following experiments are around $42 \mathrm{~mm}$ in diameter and $4 \mathrm{~m} / \mathrm{s}$ in velocity.

\section{Experimental Verification}

\subsection{Capture of Static Objects}

The grasping ability of the continuum arm group was verified firstly by grasping static objects with different sizes, shapes and stiffness. As shown in Figure 9, the capture mechanism was installed on the tip end of a KUKA robotic manipulator. Firstly, the KUKA robotic manipulator moves the capture mechanism with multiple arms to envelope the target body. Then, the linear stepper motor 1 is activated, which can simultaneously apply tension to all driving wires 1 and initiate a bending motion 

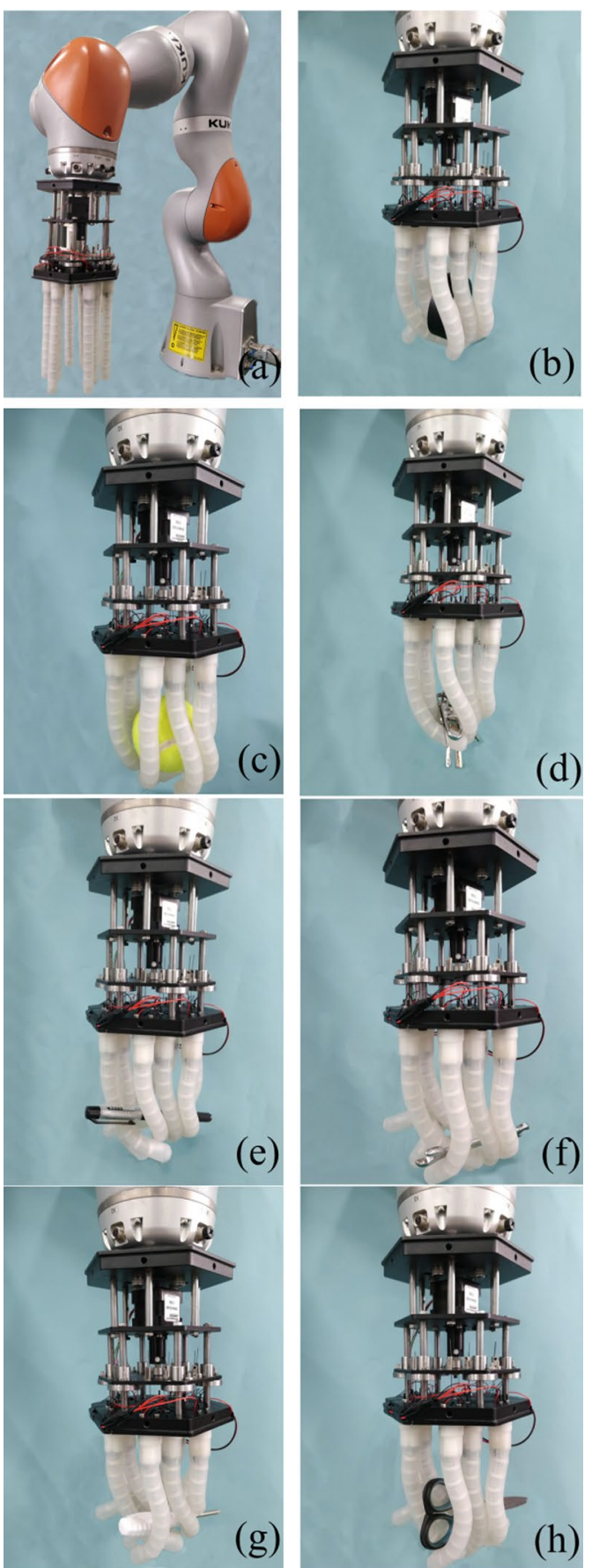

Figure 9 Universal grasp experiments: (a) KUKA robotic manipulator, (b) Tape, (c) Tennis ball, (d) Keys, (e) Pen, (f) Wrench, (g) Screwdriver, (h) Scissors

to all arms to grasp the object. Finally, the linear stepper motor 2 is activated, simultaneously applying tension to all driving wires 2 to stiffen the arms.

Seven different irregular objects including tape, tennis ball, keys, pen, wrench, screwdriver, and scissors were
Table 3 Parameters of three experiments

\begin{tabular}{|c|c|c|c|}
\hline Shape & $\begin{array}{l}\text { Incident angle of } \\
\text { the target }\left({ }^{\circ}\right)\end{array}$ & $\begin{array}{l}\text { Posture of the } \\
\text { arm group }\end{array}$ & $\begin{array}{l}\text { Success } \\
\text { rate } \\
(\%)\end{array}$ \\
\hline Ball & 0 & Upright & 90 \\
\hline $\begin{array}{l}\text { Equilateral tetra- } \\
\text { hedron }\end{array}$ & 0 & Upside-down & 82 \\
\hline Cylinder & 45 & Upright & 78 \\
\hline
\end{tabular}

tested. These objects can be successfully grasped, showing that the flexible capture mechanism inspired by sea anemone has strong adaptability to targets with various shapes and sizes.

\subsection{Dynamic Capture}

Three different shapes of targets, including a golf ball, an equilateral tetrahedron and a cylinder, were selected for the dynamic capture experiment. In addition, different incident angles of the target and postures of the arm group were utilized to demonstrate the capture adaptability, as shown in Table 3.

The dynamic capture experiment of a $42 \mathrm{~mm}$ diameter golf ball is shown in Figure 10. A spring initiated catapulting device is fabricated to launch the target. By compressing and releasing the spring in the catapulting device, the initial velocity of the target can reach $4 \mathrm{~m} / \mathrm{s}$. In Figure 10(a), The golf ball is launched to the center of the arm group at time $t_{0}$. In this time instant, the microswitch has not yet been triggered so its output stays at a high level. Motors 1 and 2 are disabled by the high level signal, as shown in Figure 10(d). When the dynamic target hits the arm group at time $t_{1}=0.04 \mathrm{~s}$, Figure 10(b), the microswitch at the arm bottom detects the vibration of the arm and changes its output from high to low, Figure 10(d). Subsequently, the motor driver 1 sends pulse signal to the stepper motor 1 and activates the arm group to envelop the target. In Figure 10(c), the stepper motor 1 reaches its maximum stroke and stops at time $t_{2}=0.2 \mathrm{~s}$, and the motor 2 begins to work, forcing the arm group to lock the target. The arm group completely locks the target body at time $t_{3}=0.31 \mathrm{~s}$.

The tests were repeated 50 times, among which the golf ball was successfully captured for 45 times. The capture success rate is $90 \%$.

We also use a 3D printed equilateral tetrahedron with side length of $40 \mathrm{~mm}$ to test the dynamic capture capability of irregular shape. In this test, we put the arm group upside-down as shown in Figure 11. It is found that the dynamic target can also be successfully captured, which 


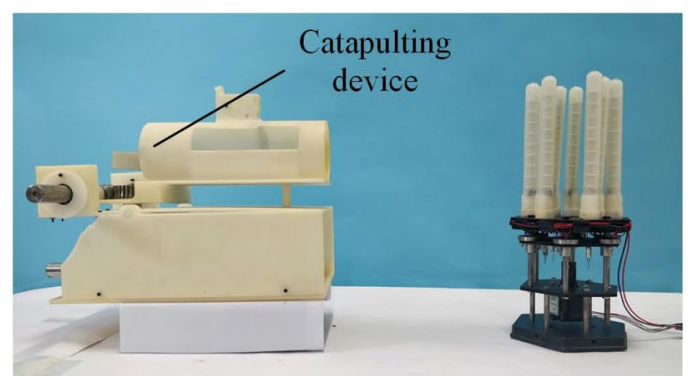

(a) The initial state of the capture mechanism $\left(t_{0}=0 \mathrm{~s}\right)$

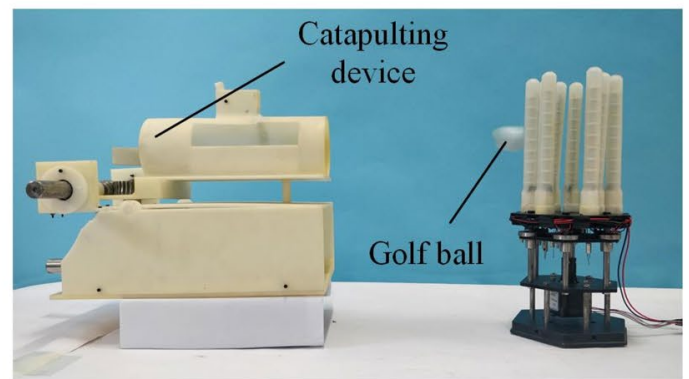

(b) The dynamic target begins to contact the arm group $\left(t_{1}=0.04 \mathrm{~s}\right)$

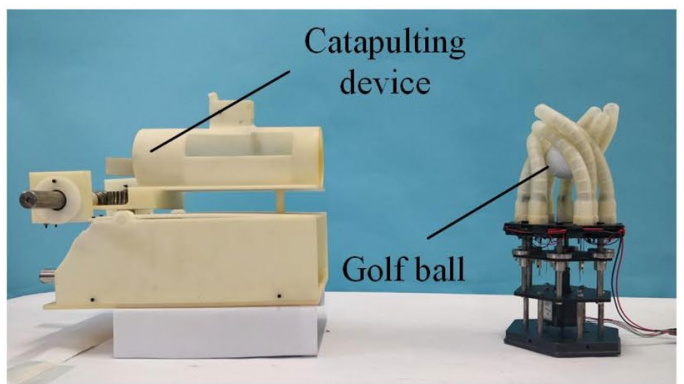

(c) The arm group completes the envelop and locking the target

$$
\left(t_{2}=0.20 \mathrm{~s}\right)
$$

\section{The electrical signal}

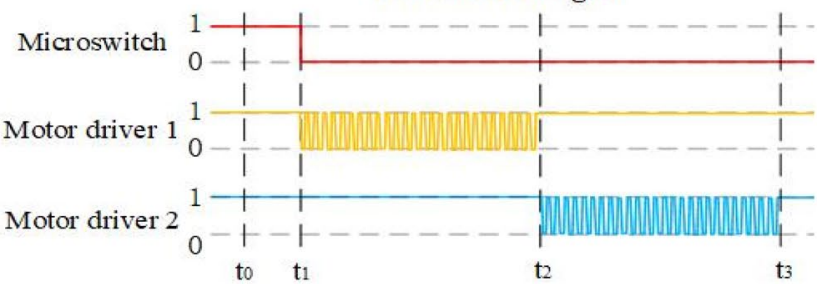

(d) Electrical signal changes during dynamic capture process

Figure 10 Dynamic capture of a golf ball

proves that the presented arm group mechanism has strong adaptability and robustness.

The tests were also carried out 50 times, in which 41 tests achieved successful capture. So the capture success rate is $82 \%$ in this case.
A cylinder with a diameter of $35 \mathrm{~mm}$ and a height of $50 \mathrm{~mm}$ was used as the dynamic target body as well. The launch angle of the catapulting device is changed from horizontal to $45^{\circ}$ upward, as shown in Figure 12. 


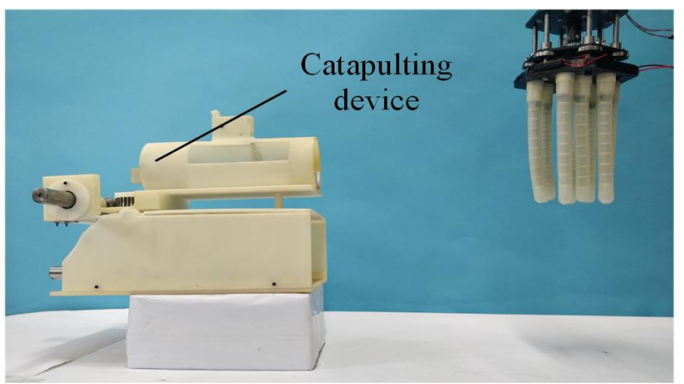

(a) The initial state of the capture mechanism $\left(t_{0}=0 \mathrm{~s}\right)$

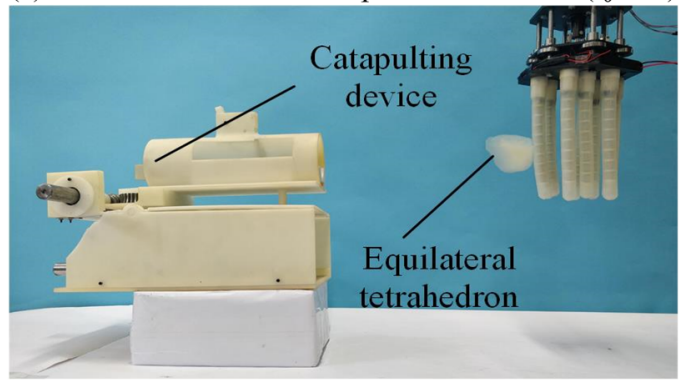

(b) The dynamic target begins to contact the arm group $\left(t_{1}=0.05 \mathrm{~s}\right)$

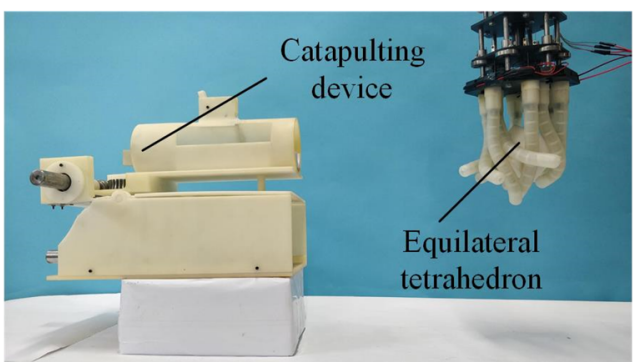

(c) The arm group completes the enveloping and locking of the target $\left(t_{2}=0.23 \mathrm{~s}\right)$

Figure 11 Dynamic capture of a tetrahedron

In this case, 39 out of 50 tests achieved successful capture. The capture success rate is $78 \%$.

\subsection{Effects of Variable Stiffness}

The tendon-driven variable stiffness mechanism makes the continuum arms achieve the combination of flexibility and load capacity. This part presents the feasibility of stiffness changes by pulling the driving wires. In our scenario, firstly, we control the linear stepper motor 1 to pull the driving wires 1 by $15 \mathrm{~mm}$ to envelop the target ball and apply an upward pulling force to the target, Figure 13. We use a dynamometer to record the maximum pulling force that the arm group can fight against to hold the target. The value of the maximum pulling force is defined as the locking force of the arm group, and is tested for 5 times as shown in Figure 14.

Then, the linear stepping motor 2 is activated to pull the driving wires 2 by $10 \mathrm{~mm}$ and therefore increases the

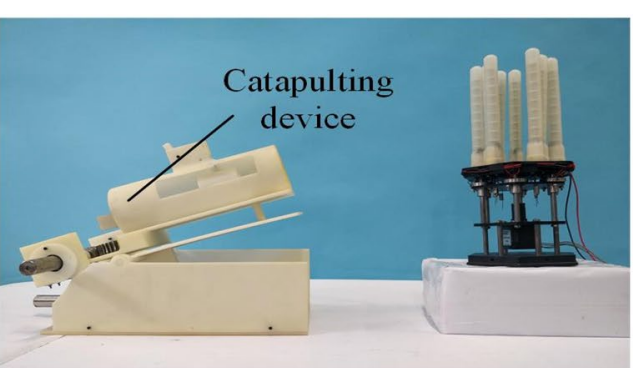

(a) The initial state of the capture mechanism $\left(t_{0}=0 \mathrm{~s}\right)$

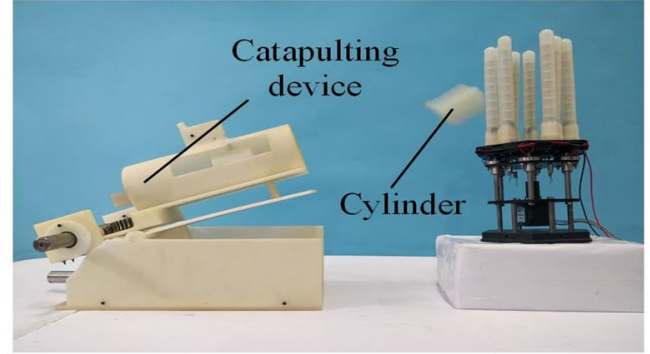

(b) The dynamic target begins to contact the arm group $\left(t_{1}=0.03 \mathrm{~s}\right)$

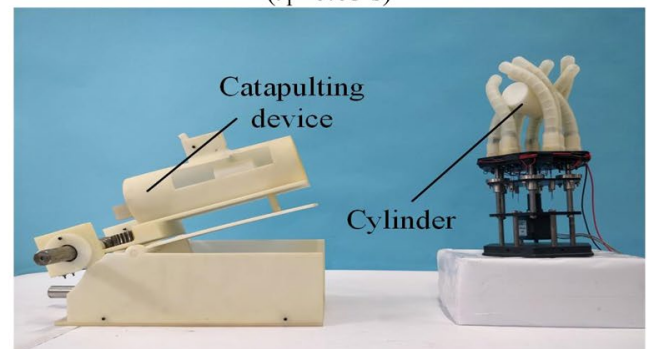

(c) The arm group completes the enveloping and locking of the target $\left(t_{2}=0.19 \mathrm{~s}\right)$

Figure 12 Dynamic capture of a cylinder

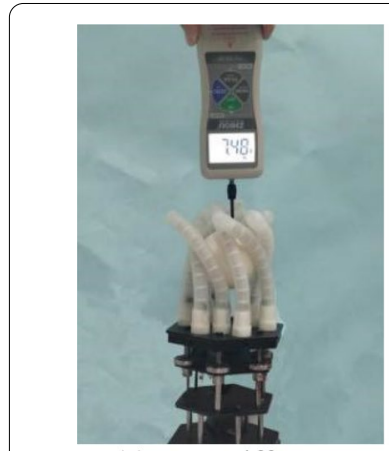

(a) Low stiffness

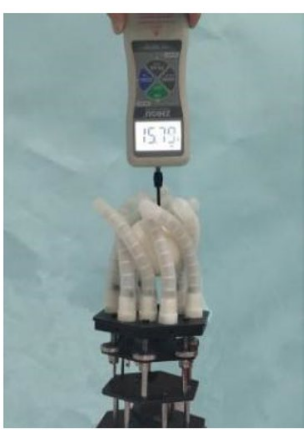

(b) High stiffness
Figure 13 Verification of variable stiffness

arm stiffness. The corresponding locking force recorded by the dynamometer are also given in Figure 14 .

It can be seen that, the average locking force is $7.32 \mathrm{~N}$ in the case of grasping with low stiffness, and will reach 


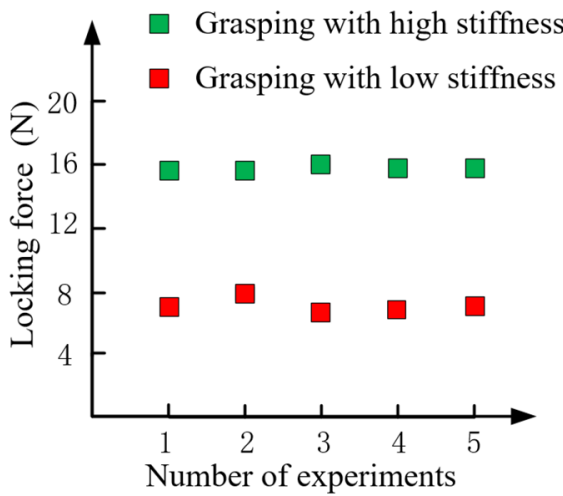

Figure 14 Locking force

$15.85 \mathrm{~N}$ when increasing the arm stiffness. In other words, the locking force of the arm group can be doubled by changing the stiffness, which will significantly help to hold the target.

\section{Conclusions}

(1) Inspired by sea anemone, a flexible capture mechanism utilizing a continuum arm group to envelop and lock non-cooperative targets is proposed. Each single arm has serially stacked segments to provide sufficient DOF to fit the shape and size of the target. Two NiTi wires are embedded along the entire length of the arm to achieve bending motion and stiffness regulation, respectively.

(2) Through finite element simulation, the influence of the arm group configuration, as well as the target size and velocity, were investigated. It is found that the capture success rate can be improved by making the clearance of arms close to the size of the target, and the distribution density of the arms should increase if the velocity of the targets increase.

(3) In addition to grasp static targets, the constructed prototype shows strong adaptability to capture dynamic targets with different sizes, shapes and incident angles. There is no need for accurate positioning and orientation control, and the average dynamic capture success rate is $83.3 \%$. The use of stiffness regulation can further increase the locking force of the mechanism.

(4) Both simulations and experiments prove that the presented mechanism can satisfy the design requirements. Future work will perform dynamic capture tests of the presented mechanism on a robotic manipulator, and investigate its potential uses for on-orbit service and underwater exploration.

\section{Authors' contributions}

JY conducted the experimental work and wrote the manuscript. CR contributed to the finite element analysis. CY and YW assisted in processing the simulated data. SW provided suggestions on experiments and conducted proof reading. RK presented the design idea and modified the manuscript. All authors read and approved the final manuscript.

\section{Authors' Information}

Jiankun Yang, born in 1996, is currently a master candidate at Key Laboratory of Mechanism Theory and Equipment Design of Ministry of Education, Tianjin University, China. His research interests include continuum and soft robotics.

Chengwei Ren, born in 1994, is currently a master candidate at Key Laboratory of Mechanism Theory and Equipment Design of Ministry of Education, Tianjin University, China. His research interests include continuum and soft robotics.

Chenghao Yang, born in 1991, is currently a PhD candidate at Key Laboratory of Mechanism Theory and Equipment Design of Ministry of Education, Tianjin University, China. His research interests include continuum and soft robotics.

Youyu Wang, born in 1982, is currently a senior engineer at Beijing Institute of Spacecraft System Engineering CAST. His research interests include aerospace engineering and mechatronic systems.

Shumin Wan, born in 1964, is currently an associate professor at Key Laboratory of Advanced Ceramics and Processing Technology of Ministry of Education, Tianjin University, China. Her research interests include automotive engineering and robotics.

Rongjie Kang, born in 1981, is currently an associate professor at Key Laboratory of Mechanism Theory and Equipment Design of Ministry of Education, Tianjin University, China. His research interests include soft robotics and mechatronic systems.

\section{Funding}

Supported by National Key R\&D Program of China (Grant Nos.

2019YFB1309800, 2018YFB1304600), National Natural Science Foundation of China (Grant No. 51875393), and State Key Laboratory of Robotics FoundationChina (Grant No. 2019-O04).

\section{Competing Interests}

The authors declare no competing financial interests.

\section{Author Details}

${ }^{1}$ Key Laboratory of Mechanism Theory and Equipment Design of Ministry of Education, Tianjin University, Tianjin 300350, China. ${ }^{2}$ State Key Laboratory of Robotics, Shenyang Institute of Automation, Chinese Academy of Sciences, Shenyang 110169, China. ${ }^{3}$ Beijing Institute of Spacecraft System Engineering CAST, Beijing 100094, China.

Received: 18 March 2020 Revised: 10 May 2021 Accepted: 5 August 2021 Published online: 21 August 2021

\section{References}

[1] FV Drigalski, D Yoshioka, W Yamazaki, et al. NAIST openhand M2S: A versatile two-finger gripper adapted for pulling and tucking textile. First IEEE International Conference on Robotic Computing (IRC). IEEE, 2017: 117-122.

[2] F C Samavati, A Feizollahi, P Sabetian, et al. Design, fabrication and control of a three-finger robotic gripper. 2011 First International Conference on Robot, Vision and Signal Processing, IEEE, 2011: 280-283.

[3] K Nie, WWan, K Harada. A hand combining two simple grippers to pick up and arrange objects for assembly. IEEE Robotics and Automation Letters, 2019, 4(2): 958-965.

[4] M Bolboe, I Staretu. Antropomorphic gripper with two symmetrical fingers, designed for industrial robots. 2014 23rd International Conference on Robotics in Alpe-Adria-Danube Region (RAAD), IEEE, 2014: 1-6.

[5] J Butterfaß, M Grebenstein, H Liu, et al. DLR-Hand II: Next generation of a dextrous robot hand. Proceedings 2001 ICRA IEEE International Conference on Robotics and Automation (Cat. No. 01CH37164), IEEE, 2001, 1: 109-114. 
[6] L U Odhner, L P Jentoft, M R Claffee, et al. A compliant, underactuated hand for robust manipulation. The International Journal of Robotics Research, 2014, 33(5): 736-752.

[7] J S Dai, D Wang, L Cui . Orientation and workspace analysis of the multifingered metamorphic hand-metahand. IEEE Transactions on Robotics, 2009, 25(4): 942-947.

[8] K Yoshida, H Nakanishi. Impedance matching in capturing a satellite by a space robot. Proceedings 2003 IEEE/RSJ International Conference on Intelligent Robots and Systems (IROS 2003)(Cat. No. 03CH37453), IEEE, 2003, 4: 3059-3064

[9] Q Meng, J Liang, O Ma. Identification of all the inertial parameters of a non-cooperative object in orbit. Aerospace Science and Technology, 2019 91: 571-582.

[10] Y W Li, Y M Bo, G P Zhao. Survey of measurement of position and pose for space non-cooperative target. 201534 th Chinese Control Conference (CCC), IEEE, Hangzhou, China, 2015: 5101-5106.

[11] I Rekleitis, E Martin, G Rouleau, et al. Autonomous capture of a tumbling satellite. Journal of Field Robotics, 2007, 24(4): 275-296.

[12] O Khatib, X Yeh, G Brantner, et al. Ocean one: A robotic avatar for oceanic discovery. IEEE Robotics \& Automation Magazine, 2016, 23(4): 20-29.

[13] M Shan, J Guo, E Gill. Review and comparison of active space debris capturing and removal methods. Progress in Aerospace Sciences, 2016, 80: 18-32.

[14] I D Walker, D M Dawson, T Flash, et al. Continuum robot arms inspired by cephalopods. Unmanned Ground Vehicle Technology VII, 2005, 5804 303-314.

[15] M W Hannan, I D Walker. The elephant trunk manipulator, design and implementation. 2001 IEEE/ASME International Conference on Advanced Intelligent Mechatronics. Proceedings (Cat. No. 01TH8556), IEEE, 2001, 1: 14-19.

[16] M Wilde, I Walker, S K Choon, et al. Using tentacle robots for capturing non-cooperative space debris-A proof of concept. AIAA SPACE and Astronautics Forum and Exposition, 2017: 5246

[17] G Zhai, B Liang, C Li. Proximity trajectory planning for net-based space robotic system. Journal of Harbin Institute of Technology, 2010, 42(09): 1356-1362.

[18] G Zhai, Y Qiu, B Liang, et al. On-orbit capture with flexible tether-net system. Acta Astronautica, 2009, 65(5-6): 613-623.
[19] B Mosadegh, P Polygerinos, C Keplinger, et al. Pneumatic networks for soft robotics that actuate rapidly. Advanced Functional Materials, 2014, 24(15): 2163-2170.

[20] F Ilievski, A D Mazzeo, R F Shepherd, et al. Soft robotics for chemists. Angewandte Chemie International Edition, 2011, 50(8): 1890-1895.

[21] K C Galloway, K P Becker, B Phillips, et al. Soft robotic grippers for biological sampling on deep reefs. Soft Robotics, 2016, 3(1): 23-33.

[22] S N Geng, YY Wang, L S Chen, et al. Design and control of a continuum arm with variable stiffness. Journal of Astronautics, 2018, 39(12): 1391-1400.

[23] C H Yang, S N Geng, I Walker, et al. Geometric constraint-based modeling and analysis of a novel continuum robot with Shape Memory Alloy initiated variable stiffness. The International Journal of Robotics Research, 2020, 39(14): 1620-1634.

[24] R J Kang, Y Guo, L S Chen, et al. Design of a pneumatic muscle based continuum robot with embedded tendons. IEEE/ASME Transactions on Mechatronics, 2017, 22(2): 751-761.

[25] X Dong, D Palmer, D Axinte, et al. In-situ repair/maintenance with a continuum robotic machine tool in confined space. Journal of Manufacturing Processes, 2019, 38(FEB.): 313-318.

[26] M F Wang, D Palmer, X Dong, et al. Design and development of a slender dual-structure continuum robot for in-situ aeroengine repair. 2018 IEEE/ RSJ International Conference on Intelligent Robots and Systems (IROS), 2018: 5648-5653.

[27] Y Li. Sea anemone: The sunflower of the deep ocean. China Nature, 2019(4): 12

[28] Q H Huang. Deflection method for measuring the elastic modulus of a metal cantilever beam. Science and Technology Information, 2011(28): 67.

[29] YT Zhang, R Ding, Z Y Zuo, et al. A new method for simultaneously measuring the elastic modulus and Poisson's ratio of a cylindrical specimen by the relationship between lateral compression force and displacement. Journal of Experimental Mechanics, 2019(3): 1.

[30] J Cui. Understanding and application of steel structure design code. China Building Industry Press, 2004. (in Chinese)

\section{Submit your manuscript to a SpringerOpen ${ }^{\circ}$ journal and benefit from:}

- Convenient online submission

- Rigorous peer review

- Open access: articles freely available online

- High visibility within the field

- Retaining the copyright to your article

Submit your next manuscript at springeropen.com 